\title{
Bacterial Infection in the Limbs of Patients with Rheumatoid Arthritis during Biological Agent Therapy ${ }^{*}$
}

\author{
Hajime Yamanaka ${ }^{\#}$, Kenichiro Goto ${ }^{\dagger}$, Munetaka Suzuki ${ }^{\dagger}$ \\ Department of Orthopaedic Surgery, National Hospital Organization Shimoshizu Hospital, Yotsukaido City, Japan. \\ Email: ${ }^{*}$ ici05323@m7.gyao.ne.jp,g10k25@yahoo.co.jp,rcjqq173@ybb.ne.jp
}

Received March 2 $2^{\text {nd }}, 2012$; revised April 12 $2^{\text {th }}, 2012$; accepted April 25 $5^{\text {th }}, 2012$

\begin{abstract}
Biological therapies in rheumatoid arthritis (RA) are known to increase the risk of serious infections. The present study was performed to evaluate the clinical features of bacterial infections occurring in the limbs during biological therapies in patients with RA. By March 2011, 11 RA patients (14 limbs) treated with biological agents at our institution required hospitalization due to bacterial infections occurring in the limbs. These patients had an average age of 53.7 years old. Infections occurred an average of 19 months after biological treatment. Two limbs in one patient were treated with infliximab, eight limbs in six patients were treated with etanercept, one limb in one patient was treated with adalimumab, and three limbs in three patients were treated with tocilizumab. Cellulitis occurred in 7 limbs, late infections after total knee arthroplasty occurred in two limbs, early infections after orthopedic surgery occurred in three limbs, and septic arthritis occurred in two limbs. Four cases had comorbidities-liver cirrhosis and diabetes mellitus in one and three cases, respectively. All patients were treated using corticosteroid with an average dose of $4.6 \mathrm{mg}$ daily. Seven limbs required surgical treatment. All patients finally recovered. Ten limbs continued treatment with biological agents. Care must be taken regarding bacterial infection in the limbs of RA patients treated by using biological agents, particularly those with comorbidities. Further studies are required to confirm means of preventing such infections in daily practice.
\end{abstract}

Keywords: Bacterial Infection; Limbs; Rheumatoid Arthritis; Biological Agents

\section{Introduction}

Rheumatoid arthritis (RA) is a chronic, progressive inflammatory disease that can lead to severe joint destruction and disability. Several cytokines, such as tumor necrosis factor (TNF) and interleukin-6 (IL-6), play critical roles in the pathogenesis of this disease [1]. Conventional treatment of RA involves a combination of corticosteroid and disease-modifying anti-rheumatic drugs (DMARDs), such as methotrexate. However, RA may remain active in some cases of treatment failure with traditional therapy. Drugs targeting these cytokines have been developed over the past few years to neutralize the precise pathways underlying the pathogenetic mechanisms of RA. At present, five different biological agents are available for use in RA in Japan, i.e., the TNF antagonists infliximab, etanercept, and adalimumab, the IL-6 receptor antagonist tocilizumab, and the T-cell costimulator modulator abatacept. Many studies have demonstrated

\footnotetext{
*Conflict of interest statement: The authors declare that they have no competing interests.

${ }^{\#}$ Corresponding author.

†Authors' contributions: K. Goto and M. Suzuki made substantial contributions to study conception and design. All authors approved the final version of this article.
}

improvements with regard in joint destruction and patient's daily life by using these biological agents [2-6]. In addition, these cytokines also play important roles in host defense [7-9]. Therefore, the use of biological drug therapy is accompanied by the need to study and understand the effects of modifying these key pathways on infection risk. The increased risk of bacterial and opportunistic infections in patients receiving biological drugs is well known [10]. Serious bacterial infections include not only tuberculosis respiratory infections, but also skin, urinary tract, bone, and joint infections. One of the more severe infections of bone and joints is surgical site infection of total joint arthroplasty (TJA) [11]. Although serious, TJA infection is rare in daily medical practice. There have been few comparative studies focusing on serious bacterial skin and bone and joint infections in RA patients receiving biological drugs. Such infections may be related to the effects of medications used to treat RA, particularly drugs that are potentially immunosuppressive.

The objectives of the present study were to retrospectively evaluate the clinical aspects of serious bacterial skin, bone, and joint infections requiring hospitalization in patients with RA receiving biological drugs.

All patients gave their informed consent prior to inclu- 
sion in the study.

\section{Patients and Methods}

A total of 273 adult RA patients who were sequential attendees at our hospital and who had an active RA were treated with four different biological agents. During the treatment period from April 2006 to March 2011, 11 patients developed bacterial infection on 14 limbs and were hospitalized at our division (Table 1). All patients fulfilled the diagnostic criteria of the American College of Rheumatology (ACR) [12]. We defined bacterial infection as septic arthritis, osteomyelitis, postoperative deep or superficial wound infection, and cellulitis, requiring hospitalization, intravenous antibiotics, or surgical therapy. Bacterial and tuberculosis pneumonia were excluded in this study. Viral infection, such as Herpes Zoster, and cases of infection after injury were also excluded. The infections were examined and diagnosed by two rheumatologists (HY, KG).

Table 1. Demographics and clinical characteristics of the patients.

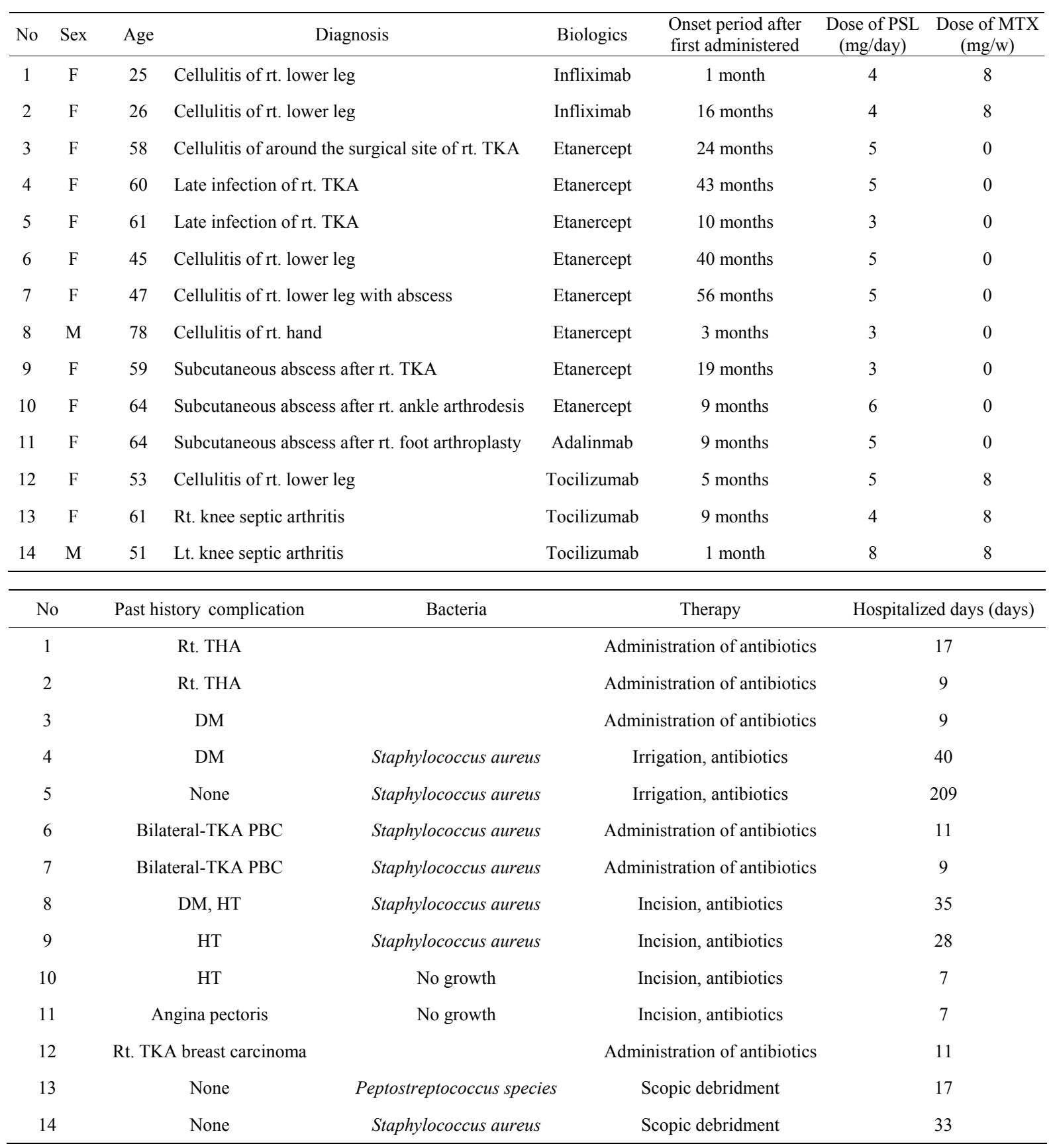

TKA: Total Knee Arthroplasty; DM: Diabetes mellitus; THA: Total Hip Arthroplasty; PBC: Primary Biliary Cirrhosis; HT: Hypertension. 
A detailed history and clinical features, including laboratory data and therapeutic method, were investigated.

\section{Results}

The 11 patients consisted of 2 men ( 2 limbs) and 9 women (12 limbs). The average age of patients was 53.7 years old (range: 25 - 78 years old), the average RA disease duration was 7.3 years (range: 1 - 18 years). The mean body mass index of the 11 patients was 25.5 (range: 20.5 - 32.3). All patients were receiving oral steroid therapy, and the average steroid dose was $4.6 \mathrm{mg}$ (range: 1 $11 \mathrm{mg}$ ) daily. Three of the 11 patients received methotrexate (MTX) therapy (dosage: $8 \mathrm{mg}$ per week in all three patients). The mean follow-up period after discharge was 3 years 4 months (range: 3 months - 5 years 4 months).

Cellulitis occurred in 5 patients (7 limbs). Six of these were lower limbs, and cellulitis occurred after TJA in all cases. The remaining case involved the upper limb. Subcutaneous infections around the surgical site early after the operation occurred on 3 patients ( 3 limbs). One of the 3 surgical operations was ankle arthrodesis and the other two were foot rejection arthroplasties with swanson implant (Wright Medical Inc., Arlington, TN). Late infections after total knee arthroplasty (TKA) occurred in two patients (two limbs). Joint infections of the knee without TJA occurred in two patients (two limbs).

With regard to the biological agents, six patients (eight limbs) received etanercept ( $25 \mathrm{mg}$ twice a week), one patient (two limbs) received infliximab $(3 \mathrm{mg} / \mathrm{kg}$, once every 8 weeks), three patients (three limbs) received tocilizumab $(8 \mathrm{mg} / \mathrm{kg}$, once every 4 weeks), and one patient (one limb) received adalimumab (40 mg once every 2 weeks).

Onset of infection occurred within one year after commencement of biological agent administration in eight limbs. However, the other cases occurred after various periods.

At the time of infection, two patients (three limbs) were also receiving treatment for diabetes mellitus and four patients (four limbs) were receiving treatment for high blood pressure. None of the patients had chronic obstructive pulmonary disorder as a comorbid condition.

Without cellulitis, we identified the bacterial species in eight limbs: Peptostreptococcus spp. in one case and Staphylococcus aureus in seven cases. However, the bacteria could not be identified in two cases, even with culture of the specimens.

About treatment, four patients (seven limbs) were cured with antibiotics only. The remaining seven patients (seven limbs) were cured by use of antibiotics and surgical treatment, such as irrigation of the wound. Finally, all patients recovered. Treatment with the same biological agent was restarted in nine patients a few months after discharge because of a worsening of RA activity due to cessation of treatment. One patient was treated with another biological agent (etanercept to tocilizumab), and one patient declined further biological agent treatment.

The average period of hospitalization was 31.6 days (range: 7 - 209 days).

\section{Typical Case Report of Bacterial Infection on the Limb}

A 47-year-old woman was diagnosed with RA and primary biliary cirrhosis (PBC) in 1999. Blood analysis indicated pancytopenia due to $\mathrm{PBC}$. She underwent right TKA in 2008. As treatment with infliximab was ineffective, she was administered etanercept at a dose of $25 \mathrm{mg}$ twice a week from February 2009. She developed right lower leg cellulitis suddenly in April 2010 and treatment with antibiotic (sulbactam/piperacillin; SBT/ABPC, $3 \mathrm{~g} /$ day) about two weeks after hospitalization, which finally healed. However, in July of the same year, she developed lower right cellulitis again with several abscesses (Figures 1(a) and (b)). Blood examination showed a C-reactive protein level of $4.9 \mathrm{dl} / \mathrm{ml}$ and white blood cell count of $1900 / \mu l$. Surgical treatment was performed with incision of the abscess and SBT/ABPC was administered at 3 $\mathrm{g} /$ day for 2 weeks. The patient was finally healed about 1 month after hospitalization. Pus from the abscess was cultured, and showed growth of Staphylococcus aureus.

\section{Discussion}

In this report, we presented 11 patients (14 limbs) with RA receiving biological treatment experiencing the onset of skin, bone, and joint bacterial infections. The majority were patients with infectious lesions typical of acute inflammatory

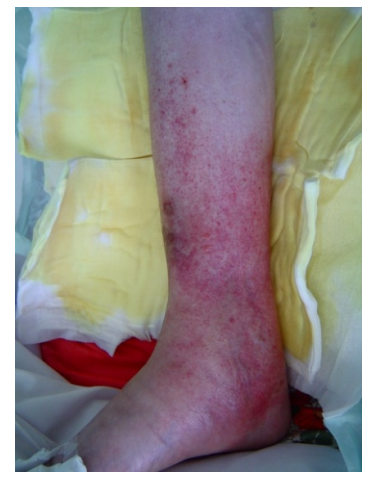

(a)

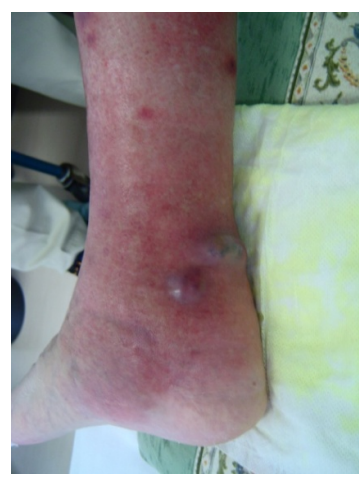

(b)
Figure 1. Clinical appearance of the right leg. (a) Anterior to the medial aspect of the right leg. Erythema with multiple, small subcutaneous hemorrhages were observed; (b) Medial aspect of the right leg. Several subcutaneous abscesses were observed. 
diseases, but various clinical patterns were observed. These infections appear as rare but potentially severe complications of biological therapies.

RA patients are often treated with immunosuppressive drugs, such as glucocorticoids and MTX that contribute to the risk of infection. However, there is evidence that the underlying disease itself, the activity of the disease, and the degree of disability are independent risk factors in addition to immunosuppressive treatment. TNF and IL-6 are pleiotropic proinflammatory cytokines and play central roles in the pathogenesis of rheumatic diseases. These cytokine blockers have well-established efficacy in treatment of RA patients. However, the risk of serious infections in patients with RA treated with anti-TNF blockers was double that in patients administered placebo as controls [13]. Galloway [14] reported that anti-TNF therapy increased the risk of serious infections especially in the first 3 to 6 months of treatment.

However, less is known about the clinical features and incidence rates of bacterial infection in the limbs including postoperative infections, skin, and bone-joint infections.

Some reports [15-17] indicated a significant association between early infectious complications following orthopedic surgery and treatment with TNF inhibitors in patients with RA. Especially in TJA surgical site infection, important risk factors are primary or revision TJA within the last year, particularly when TNF blockers are not interrupted before surgery, and a daily steroid intake over $5 \mathrm{mg}$ [18].

The incidence rates of bacterial infection on the limbs associated with postoperative infections, skin, and bonejoint infections in patients with RA receiving biological agents were 2.76 to 7.68 times higher than in those without biological treatment $[19,20]$.

However, there have been no reports regarding the detailed mechanisms underlying these increased rates of infection. Several mechanisms have been postulated, including disturbance of important functions of various cells in the skin by TNF and IL-6 inhibitors [21]; increased sensitivity and reactivity of the skin to bacteria [22]; and disruption of normal wound healing reaction [23]. Moreover, complication with diabetes mellitus or respiratory disease affected patient's immune condition. Skin condition was very poor in patients using steroids. However, the details remain unclear.

Previous studies were performed to examine differences in the incidence rates of infection between biological agents, but no differences were identified [23-25]. Five different biological agents are approved for use in RA therapy in Japan. In the present study, the number of cases of infection was higher among patients treated with etanercept than in those given the other agents. However, we could not conclude that etanercept is associated with serious infection due to the small number of patients included in this study.

Diabetes mellitus and steroid intake have previously been shown to be associated with increased risk of infection in RA $[24,25]$. In this study, only two patients (three limbs) were accompanied with diabetes mellitus, and although all patients had an average corticosteroid dose of $4.6 \mathrm{mg}$ daily, this dose was not particularly high. We did not identify individual risk factors in this study. The small number of cases included in this study did not permit confirmation of hypotheses regarding causative relationships.

How should we guard against infectious diseases in the limbs of RA patients during biological drug use? It is unlikely that we can completely prevent infections in these cases. However, it is important to inform patients that infection on the limbs is not unusual, and to take steps to prevent such infection by skin care, avoiding excessive load on the limbs in daily living, to go immediately to hospital if signs of infection appear on the limb, etc. Medication for diabetes mellitus or respiratory disease is very important. Furthermore, it is necessary to avoid worsening the infection by consideration of surgical care immediately and patients with infectious symptoms may be hospitalized.

There were several limitations of our study. First, this was a retrospective study, and the number of patients was small. The small number of cases limited the statistical power of the study. Second, diagnosis is the most important issue. Infection was diagnosed by blood analysis, skin condition, bacteria culture analysis, etc. However, it was difficult to confirm the relation between administration of biological agents and infection. Further studies are required to clarify these points. Third, some potential risk factors (recent pulmonary infection, number of previous disease-modifying anti-rheumatic drugs (DMARDs), cumulative steroid intake, non-steroidal anti-inflammatory drugs) were not assessed because almost all patients had already been treated with RA at other hospitals before starting treatment with biological agents. Fourth, this was not a cohort and randomized control study. Our analyses were based on a limited number of episodes of limb infection. Fifth, our study did not include possible cases of mild infections that did not require hospitalization, and so may have underestimated the incidence rate.

In summary, we have demonstrated a significant complication of serious bacterial infection on the limbs of RA patients treated with biological agents. Our overall results raise concerns about how best to balance safety with effectiveness. Patients and physicians - both rheumatologists and orthopedic surgeons - must emphasize the elevated risk for awareness and vigilantly monitor for signs of infection. These findings support the need for additional investigations and development of clinical practices to 
prevent infection on the limbs in RA patients.

\section{Conclusion}

In RA patients receiving biological agents, serious bacterial infection on the limbs is rare but potentially severe complication. Physicians should exercise caution and continue to be monitored to these infections during biological agent therapy in RA patients.

\section{REFERENCES}

[1] D. L. Scott, K. A. Grindulis, G. R. Struthers, B. L. Coulton, A. L. Popert and P. A. Bacon, "Progression of Radiological Changes in Rheumatoid Arthritis," Annals of the Rheumatic Diseases, Vol. 43, No. 1, 1984, pp. 8-17. doi:10.1136/ard.43.1.8

[2] T. Takeuchi, H. Yamanaka, E. Inoue, H. Nagasawa, M. Nawata, K. Ikari, K. Saito, N. Sekiguchi, E. Sato, H. Kameda, S. Iwata, T. Mochizuki, K. Amano and Y. Tanaka, "Retrospective Clinical Study on the Notable Efficacy and Related Factors of Infliximab Therapy in a Rheumatoid Arthritis Management Group in Japan: One-Year Outcome of Joint Destruction (RECONFIRM-2J)," Modern Rheumatology, Vol. 18, No. 5, 2008, pp. 447-452. doi:10.1007/s10165-008-0077-5

[3] L. Klareskog, D. van der Heijde, J. P. de Jager, A. Gough, J. Kalden, M. Malaise, M. E. Martin, K. Pavelka, J. Sany, L. Settas, J. Wajdula, R. Pedersen, S. Fatenejad, M. Sanda, et al., "Therapeutic Effect of the Combination of Etanercept and Methotrexate Compared with Each Treatment Alone in Patients with Rheumatoid Arthritis: DoubleBlind Randomized Controlled Trial," Lancet, Vol. 363, No. 9410, 2004, pp. 675-681. doi:10.1016/S0140-6736(04)15640-7

[4] F. C. Breedveld, M. H. Weisman, A. F. Kavanaugh, S. B. Cohen, K. Pavelka and R. van Vollenhoven, "The PREMIER Study: A Multicenter, Randomized, Double-Blind Clinical Trial of Combination Therapy with Adalimumab Plus Methotrexate versus Methotrexate Alone or Adalimumab Alone in Patients with Early, Aggressive Rheumatoid Arthritis Who Had Not Had Previous Methotrexate Treatment," Arthritis Care \& Research, Vol. 54, No. 1, 2006, pp. 26-37. doi:10.1002/art.21519

[5] N. Nishimoto and N. Takagi, "Assessment of the Validity of the 28-Joint Disease Activity Score Using Erythrocyte Sedimentation Rate (DAS28-ESR) As a Disease Activity Index of Rheumatoid Arthritis in the Efficacy Evaluation of 24-Week Treatment with Tocilizumab: Subanalysis of the SATORI Study," Modern Rheumatology, Vol. 20, No. 6, 2010, pp. 539-547. doi:10.1007/s10165-010-0328-0

[6] M. Schiff, "Abatacept Treatment for Rheumatoid Arthritis," Rheumatology, Vol. 50, No. 3, 2011, pp. 437-449. doi:10.1093/rheumatology/keq287

[7] G. Camussi, E. Albano, C. Tetta and F. Bussolio, "The Molecular Action of Tumor Necrosis Factor-Alpha," European Journal of Biochemistry, Vol. 202, No. 1, 1991, pp. 3-14.

[8] F. M. Brennan and I. B. McInnes, "Evidence That Cyto- kines Play a Role in Rheumatoid Arthritis," Journal of Clinical Investigation, Vol. 118, No. 11, 2008, pp. 35373545. doi:10.1172/JCI36389

[9] P. Emery, P. Durez and M. Dougados, "The Impact of T-Cell Co-Stimulation Modulation in Patients with Undifferentiated Inflammatory Arthritis or Very Early Rheumatoid Arthritis: A Clinical and Imaging Study of Abatacept," Annals of the Rheumatic Diseases, Vol. 69, No. 3, 2010, pp. 510-516. doi:10.1136/ard.2009.119016

[10] J. Listing, A. Strangfeld, S. Kary, R. Rau, U. von Hinueber, M. Stoyanova-Scholz, E. Gromnica-Ihle, C. Anatoni, P. Herzer, J. Kekow, M. Schneider and A. Zink, "Infections in Patients with Rheumatoid Arthritis Treated with Biologic Agents," Arthritis Care \& Research, Vol. 52, No. 11, 2005, pp. 3403-3412. doi:10.1002/art.21386

[11] M. Gilson, L. Gossec, X. Mariette, D. Gherissi, M. H. Guyot, J. M. Berthelot, D. Wendiling, C. Michelet, P. Dellamonica, F. Tubach, M. Dougados and D. Salmon, "Risk Factors for Total Joint Arthroplasty Infection in Patients Receiving Tumor Necrosis Factor $\alpha$-Blockers: A Case-Control Study," Arthritis Research \& Therapy, Vol. 12, No. 4, 2010, p. R145. doi:10.1186/ar3087

[12] F. C. Arnett, S. M. Edworthy, D. A. Bloch, D. J. McShane, J. F. Fries, N. S. Cooper, L. A. Healey, S. R. Kaplan, M. H. Liang, H. S. Luthra, et al., "The American Rheumatism Association 1987 Revised Criteria for the Classification of Rheumatoid Arthritis," Arthritis Care \& Research, Vol. 31, No. 3, 1988, pp. 315-324. doi:10.1002/art.1780310302

[13] W. G. Dixon, K. Watson, M. Lunt, L. Hyrich, et al., "Rates of Serious Infection, Including Site-Specific and Bacterial Intracellular Infection, in Rheumatoid Arthritis Patients Receiving Anti-Tumor Necrosis Factor Therapy," Arthritis Care \& Research, Vol. 54, No. 8, 2006, pp. 2368-2376. doi:10.1002/art.21978

[14] J. B. Galloway, K. L. Hyrich, L. K. Mercer, W. G. Dixon, B. Fu, A. P. Ustianowski, K. D. Watson, M. Lunt, et al., "Anti-TNF Therapy Is Associated with an Increased Risk of Serious Infections in Patients with Rheumatoid Arthritis Especially in the First 6 Months of Treatment: Updated Results from the British Society for Rheumatology Biologics Register with Special Emphasis on Risks in the Elderly," Rheumatology, Vol. 50, No. 1, 2011, pp. 124131. doi:10.1093/rheumatology/keq242

[15] J. T. Giles, S. J. Bartlett, A. C. Gelber, S. Nanda, K. Fontaine, V. Ruffing and J. Bathon, "Tumor Necrosis Factor Inhibitor Therapy and Risk of Serious Postoperative Orthopedic Infection in Rheumatoid Arthritis," Arthritis Care \& Research, Vol. 55, No. 2, 2006, pp. 333-337. doi:10.1002/art.21841

[16] C. Bibbo and J. W. Goldberg, "Infectious and Healing Complications after Elective Orthopaedic Foot and Ankle Surgery during Tumor Necrosis Factor-Alpha Inhibition Therapy," Foot Ankle International, Vol. 25, No. 5, 2004, pp. 331-335.

[17] A. A. Broeder, M. C. Creemers, J. Fransen, E. D. Jong, D. J. R. D. Rooij, A. Wymenga, M. D. Waal-Malefijt and F. H. J. V. D. Hoogen, "Risk Factors for Surgical Site Infections and Other Complications in Elective Surgery in Patients with Rheumatoid Arthritis with Special Attention 
for Anti-Tumor Necrosis Factor: A Large Retrospective Study," Journal of Rheumatology, Vol. 34, No. 4, 2007, pp. 653-655.

[18] A. Strangfeld and J. Listing, "Bacterial and Opportunistic Infections during Anti-TNF Therapy," Best Practice \& Research Clinical Rheumatology, Vol. 20, No. 6, 2006, pp. 1181-1195. doi:10.1016/j.berh.2006.08.010

[19] T. Bongarts, A. Sutton, M. Sweeting, I. Buchan, E. Matteson and V. Montori, "Anti-TNF Antibody Therapy in Rheumatoid Arthritis and the Risk of Serious Infections and Malignancies," Journal of the American Medical Association, Vol. 295, No. 19, 2006, pp. 2275-2285.

[20] J. D. Greenberg, G. Reed, J. M. Kremer, E. Tindall, A. Kavanaugh, C. Zheng, W. Bishai, M. C. Hochberg, et al., "Association of Methotrexate and Tumor Necrosis Factor Antagonists with Risk of Infectious Outcomes Including Opportunistic Infections in the CORRONA Registry," Annals of the Rheumatic Diseases, Vol. 69, No. 2, 2008, pp. 380-386. doi:10.1136/ard.2008.089276

[21] H. H. Lee, I. H. Song and M. Friedrich, "Cutaneous Side-Effects in Patients with Rheumatoid Diseases during Application of Tumor Necrosis Factor- $\alpha$ Antagonists," British Journal of Dermatology, Vol. 156, No. 3, 2007, pp. 486-491. doi:10.1111/j.1365-2133.2007.07682.x

[22] C. Vestergaard, C. Johansen, K. Otkjaer, M. Deleuran and L. Iversen, "Tumor Necrosis Factor-Alpha-Induced CTACK/ CCL27 (Cutaneous T-Cell-Attracting Chemokine) Production in Keratinocytes Is Controlled by Nuclear Factor Kappa B," Cytokine, Vol. 29, No. 2, 2005, pp. 49-55. doi:10.1016/j.cyto.2004.09.008

[23] J. A. Hamilton and P. P. Tak, "The Dynamics of Macrophage Lineage Populations in Inflammatory and Autoimmune Disease," Arthritis \& Rheumatism, Vol. 60, No. 5, 2009 1210-1221. doi:10.1002/art.24505

[24] D. M. Grennan, J. Gray, J. Loudon and S. Fear, "Methotrexate and Early Postoperative Complications in $\mathrm{Pa}$ tients with Rheumatoid Arthritis Undergoing Elective Orthopaedic Surgery," Annals of the Rheumatic Diseases, Vol. 60, No. 3, 2001, pp. 214-217. doi:10.1136/ard.60.3.214

[25] D. Lacaille, D. P. Guh, M. Abarahamowicz, A. H. Anis and J. M. Esdaile, "Use of Nonbiologic Disease-Modifying Anti-Rheumatic Drugs and Risk of Infection in Patients with Rheumatoid Arthritis," Arthritis Care \& Research, Vol. 59, No. 8, 2008, pp. 1074-1081. doi:10.1002/art.23913 